\title{
Therapeutic Potential of Human Nasal Inferior Turbinate-Derived Stem Cells: Microarray Analysis of Multilineage Differentiation
}

\author{
Sun Hwa Park a, bo Hyun Kimª, Mi Hyun Limª,b Sang A. Back ${ }^{a}$ \\ Byeong Gon Yun ${ }^{a, b}$ Jung Ho Jeun ${ }^{a, b}$ Jung Yeon Limª, bu Young Kim ${ }^{c}$ \\ Se Hwan Hwang ${ }^{a}$ Sung Won Kim ${ }^{a}$ \\ aDepartment of Otolaryngology-Head and Neck Surgery, College of Medicine, The Catholic University of Korea,

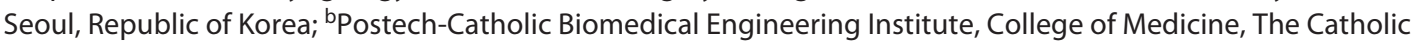 \\ University of Korea, Seoul, Republic of Korea; 'Department of Pathology, College of Medicine, The Catholic \\ University of Korea, Seoul, Republic of Korea
}

\section{Keywords \\ Human stem cells - Differentiation - Osteogenesis . Chondrogenesis · Neurogenesis · Gene expression . Microarrays $\cdot$ Gene ontology terms}

\begin{abstract}
Introduction: Human nasal inferior turbinate-derived stem cells (hNTSCs) are attractive sources of adult stem cells for medical application because they can be easily obtained and cultivated with a highly proliferative capacity. The ability of hNTSCs to differentiate into chondrocytes, osteocytes, and neural cells makes them potential replacement therapeutic candidates in intractable disease. Nevertheless, detailed expression pattern of genes associated with trilineage differentiation (osteogenesis, chondrogenesis, and neurogenesis) in hNTSCs has not been revealed yet. Methods: In this study, we aimed to evaluate gene expression patterns of various transcription factors and marker genes associated with a particular lineage (osteogenesis, chondrogenesis, and neurogenesis) of differentiation of hNTSCs by DNA microarrays. Results: In microarrays, 36 transcripts such as E2F transcription factor 1, activating transcription factor 5, and AKR1B10 were upregulated and 36 transcripts such as CA9, PPFIA4,
\end{abstract}

HAS2, and COL4A4 were downregulated in osteogenically differentiated hNTSCs. In chondrogenically differentiated hNTSCs, 3 transcripts (NUDT14, CPA4, and heparin-binding epidermal growth factor-like growth factor) were upregulated and 82 transcripts such as PTGS1, CLEC2D, and TET1 were downregulated. In neurally differentiated hNTSCs, 61 transcripts such as insulin-like growth factor-binding protein-1, nerve growth factor receptor, FGF1, OLFML1, and EPGN were upregulated and 98 transcripts such as ACAN, RUNX2, and C21 orf96 were downregulated. In gene ontology (GO) analysis, cell signal-related GO terms were highly expressed. By contrast, catalysis $\mathrm{GO}$ terms and $\mathrm{GO}$ terms related to oxidoreductase were overrepresented in chondrogenically differentiated hNTSCs and osteogenically differentiated hNTSCs, respectively. Conclusion: Considering overall results, hNTSCs-specific genetic information may promote further studies on intracellular mechanisms defining key features of these cells.

(C) 2021 The Author(s) Published by S. Karger AG, Basel

Se Hwan Hwang and Sung Won Kim also contributed equally to this work as corresponding authors.
(C) 2021 The Author(s)

Published by S. Karger AG, Basel

This is an Open Access article licensed under the Creative Commons Attribution-NonCommercial-4.0 International License (CC BY-NC) (http://www.karger.com/Services/OpenAccessLicense), applicable to the online version of the article only. Usage and distribution for commercial purposes requires written permission.
Correspondence to:

Se Hwan Hwang, yellobird@ catholic.ac.kr

Sung Won Kim, kswent@ catholic.ac.kr 


\section{Introduction}

Mesenchymal stem cells (MSCs) are adult stem cells with the potential to differentiate into various cells lineages [1]. They can be isolated from various tissues of adults $[1,2]$. Previously, we have shown that MSCs derived from nasal mucosa (inferior turbinate) (hNTSCs) have a high proliferation potential. In addition, characteristics of hNTSCs do not change regardless of cell passage number [1]. Moreover, hNTSCs show superior potential to differentiate into osteocytes and chondrocytes. These cells have better osteogenic potential regardless of accessorial osteogenic factors, including vitamin D3 and BMP-2, in comparison with MSCs isolated from bone marrow or adipose tissues [3]. These cells can differentiate easily into chondrocytes by in vitro culture without co-culture of MSCs and chondrocytes which can enhance matrix deposition [4]. MSCs are considered as important sources for cellular therapy due to their immunologically privileged properties. Additionally, the isolation of MSCs from tissues normally removed during surgery and storage of them would have significant advantage for patients with various diseases [5]. Although hNTSCs provide a novel opportunity for regenerative medicine, in otorhinolaryngologic parts, core genes during the differentiation process of hNTSCs have not been evaluated sufficiently. Thus, the objective of this study was to determine differentiation phenotypes of hNTSCs under appropriate differentiation-induction media (osteogenic, chondrogenic, and neurogenic differentiation) and perform microarray-based gene expression profiling to evaluate these differentiation phenotypes of cell populations.

\section{Materials and Methods}

\section{Statement of Ethics}

hNTSCs were isolated from patients who underwent an inferior turbinate surgery. Isolation procedures were approved by the Institutional Review Board of Seoul St. Mary's Hospital, the Catholic University of Korea (KC08TISS0341). Written informed consent was obtained from the subjects.

\section{Cell Isolation}

The inferior turbinate tissue obtained after surgery was washed 3 to 5 times with an antibiotic-antimycotic solution (Gibco, Gaithersburg, MD, USA) and 3 times with phosphate buffered saline (PBS). It was then cut into $0.5-\mathrm{mm}^{3}$ pieces. These pieces were placed into a culture dish. The dish was covered with a sterilized glass cover slide. Alpha-MEM (WELGENE, Gyeongsan, Korea) containing $10 \%$ fetal bovine serum (FBS; WELGENE) and 1\% antibiotic-antimycotic solution (Gibco) was added. It was changed every 2 days. After 15 days of culture at $37^{\circ} \mathrm{C}$ in a $5 \% \mathrm{CO}_{2}$ incuba- tor, the glass cover slide was removed and tissues floating in the culture media were removed by washing. hNTSCs attached to the bottom of the culture dish were then detached using $1 \mathrm{~mL}$ of $0.05 \%$ trypsin in $1 \mathrm{mM}$ EDTA. After isolation, hTMSCs were seeded at an initial cell density of $1 \times 10^{4}$ cells $/ \mathrm{cm}^{2}$ and amplified in a monolayer culture media. hNTSCs were cultured for 3 passages to evaluate their multidifferentiation potential.

\section{Characterization of Surface Markers on hNTSCs by Flow} Cytometry

To investigate the expression of cell surface maker proteins in hNTSCs at passage 3, cells were detached and labeled with FITCor PE-coupled anti-human antibodies (CD14, CD19, CD29, CD34, CD73, CD90, and HLA-DR; BD Biosciences, San Jose, CA, USA). Ten thousand labeled cells were measured using a FACS calibur flow cytometer (Becton Dickinson, Franklin lakes, NJ, USA). Results were analyzed with a CellQuest software (Becton Dickinson).

\section{Multilineage Differentiation Potential of hNTSCs}

To investigate the osteogenic potential of hNTSCs, cells were cultured in a low-glucose DMEM supplemented with $10 \%(\mathrm{v} / \mathrm{v})$ FBS, $0.1 \mu \mathrm{M}$ dexamethasone, $10 \mathrm{mM} \beta$-glycerophosphate, $50 \mu \mathrm{M}$ ascorbate-2-phosphate, $100 \mathrm{U} / \mathrm{mL}$ penicillin, and $100 \mu \mathrm{g} / \mathrm{mL}$ streptomycin. Culture media were changed twice every week for 4 weeks. Differentiation to osteogenic lineages was induced according to the procedures described by Hwang et al. [1]. A 3-dimensional culture system was used for chondrogenic differentiation with open-cell polylactic acid (BD Biosciences). After $2 \times 10^{6}$ cells were seeded onto the scaffold, cells were cultured in chondrogenic induction media consisting of DMEM supplemented with $10 \%$ FBS, $100 \mathrm{nM}$ dexamethasone, $50 \mu \mathrm{M}$ ascorbic acid-2 phosphate (Sigma, St. Louis, MO, USA), $50 \mathrm{mg} / \mathrm{mL}$ insulin-transferrin-sodium selenite, $1 \mathrm{mM}$ sodium pyruvate (Gibco), $40 \mu \mathrm{M}$ proline, and 2 mML-glutamine added to chondrogenic supplements TGF- $\beta 1$ (10 $\mathrm{ng} / \mathrm{mL}$; Sigma) and IGF-1 (10 ng/mL; Sigma). Culture media were changed twice every week for 2 weeks. To induce neurogenic differentiation, hNTSCs were seeded onto 4 -well chamber slides $(1 \times$ $10^{4}$ cells/well; Nalgene Nunc International, Rochester, NY, USA) and cultivated in neurobasal media. Cultivated cells were resuspended in DMEM/F-12 (Gibco) with $1 \mathrm{~mL}$ B-27 supplement, neurogenic supplements $(10 \mathrm{ng} / \mathrm{mL}$ glial-derived neurotrophin factor [Invitrogen, Carlsbad, CA, USA], $10 \mathrm{ng} / \mathrm{mL}$ neurotrophin-3 [Invitrogen], $10 \mathrm{ng} / \mathrm{mL}$ brain-derived neurotrophin factor [Invitrogen], 2 mML-glutamine), $0.5 \%$ heparin, and $1 \%$ penicillin-streptomycin (Gibco) for 2 weeks. Culture medium was refreshed every 2 days.

\section{Immunocytochemistry of Differentiated hNTSCs}

The differentiated hNTSCs were fixed with $4 \%$ paraformaldehyde in PBS for $20 \mathrm{~min}$, and nonspecific binding sites were blocked by a 30 -min incubation in PBS containing $0.5 \%$ Triton X-100 (Promega Co. Medison, WI, USA) at room temperature, then stained to analyze cytologically with Safranin O and Alizarin red for chondrocyte and osteocyte differentiation. For immunocytofluorescence staining, it is followed by antigen retrieval with proteinase $\mathrm{K}$ (Abcam) to expose the antigenic sites. After blocking with 5\% normal goat serum, cells were each incubated with primary antibodies against NeuN (Abcam) and $\beta$-tubulin (Abcam) at room temperature overnight for double staining and washed triply with 0.01 $\mathrm{mol} / \mathrm{L}$ PBS. After incubating with secondary antibodies, Alexa 488 


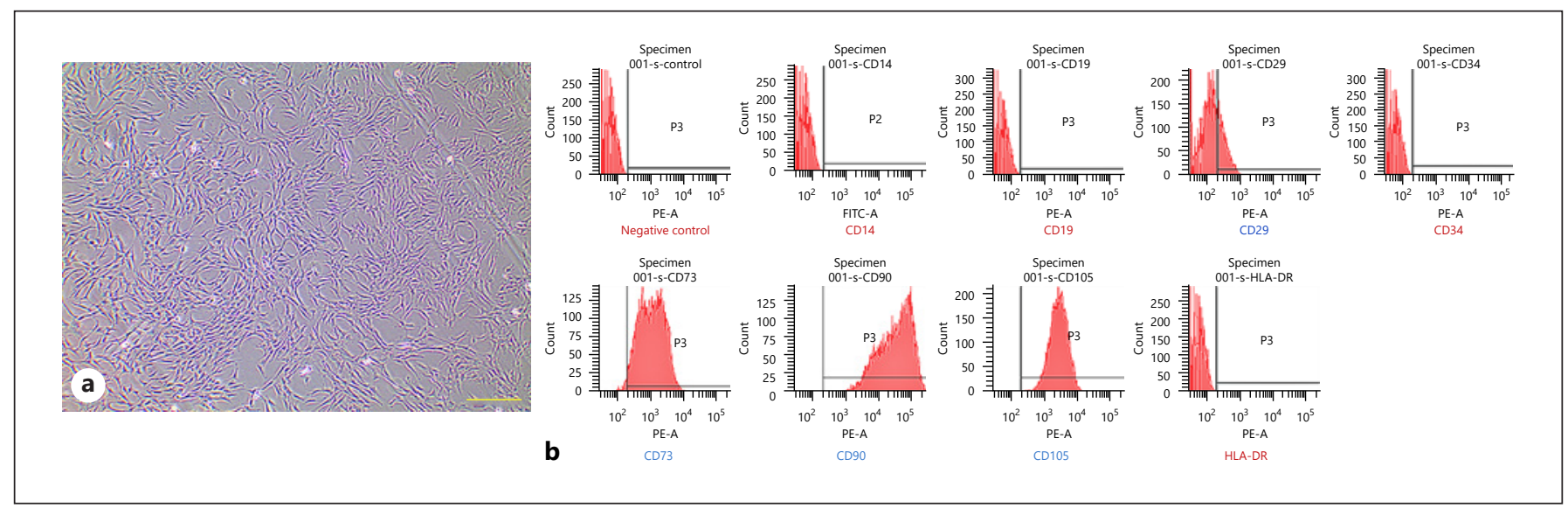

Fig. 1. a FACS analysis of hTMSCs 2 weeks after primary explant culture. The hTMSCs did not show the hematopoietic lineage markers but expressed mesenchymal stem cell markers (after 3 passages). CD14, CD19, and CD34 are markers of hematopoietic stem cells. CD29, CD73, and CD90 are markers of MSCs. HLA-DR is a human leukocyte antigen (b). FACS, fluorescence-activated cell sorting; hTMSCs, human turbinate mesenchymal stromal cells; MSCs, mesenchymal stem cells.
$(1: 100)$ and Alexa $546(1: 100)$ at room temperature for $2 \mathrm{~h}$, antigen expression was each identified. For staining nuclei, cells were mounted with mounting medium (Vectashield, Burlingame, CA, USA) conjugated with DAPI and viewed using a fluorescence-attached microscope (OlympusAX70TR62A02, Tokyo, Japan).

RNA Extraction from hNTSCs and Microarray-Based Global Gene Expression

Total RNA was extracted from cells cultured under different induction media for chondrogenic, osteogenic, or neurogenic differentiation with an RNA isolation kit (Kontes, Vineland, NJ, USA). Cy3-labeled cDNA was synthesized using $200 \mathrm{ng}$ of total RNA with a Low Input Quick Amp Labeling Kit (Agilent Technologies, Santa Clara, CA, USA). cRNAs were hybridized on a Custom Gene Expression Microarray, $4 \times 44 \mathrm{k}$ (Agilent Technologies) at $65^{\circ} \mathrm{C}$ for $17 \mathrm{~h}$. After that, microarrays were processed in accordance with the manufacturer's recommended protocol. We applied quantile normalization to compensate interarray variations. Genes with little variation and those with entropy values less than the 10th percentile were excluded. The genes were analyzed for significance by selecting 100 genes with the most significant differences by the SAM method. There are genes with the same significance (delta value), so the specific number is slightly different.

\section{Results}

\section{Isolation and Characterization of hNTSCs through Flow Cytometry}

Fibroblast-like cells appeared within 3 days, adhered to the culture dish, and formed into a single layer. Flow cytometric analysis was performed for cell surface markers of in vitro-cultured hNTSCs. Results revealed that these cells did not show any expression of hematopoietic cell markers (CD14, CD19, CD34, or HLA-DR). How- ever, they showed expression of MSC markers (CD29, CD73, and CD90) (Fig. 1).

\section{Immunocytochemistry of Differentiated hNTSCs}

The cells exposed to osteogenic medium showed direct evidences of calcium mineralization seen by Alizarin Red staining. Cells after neurogenic induction for 14 days were analyzed with immunocytochemistry and the expression of nestin and $\beta$-tubulin was detected. Safranin O binds specifically detected the proteoglycan in chondrogenic differentiated hNTSCs (Fig. 2).

\section{Gene Expression Profile}

In osteogenic differentiated hNTSCs, 36 transcripts such as E2F transcription factor 1 (E2F1), activating transcription factor 5 (ATF5), and AKR1B10 were upregulated and 36 transcripts such as CA9, PPFIA4, HAS2, and COL4A4 were downregulated. In chondrogenic differentiated hNTSCs, 3 transcripts (NUDT14, CPA4, and heparin-binding epidermal growth factor-like growth factor [HBEGF]) were upregulated and 82 transcripts such as PTGS1, CLEC2D, and TET1 were downregulated. In neural differentiated hNTSCs, 61 transcripts such as insulinlike growth factor-binding protein (IGFBP)-1, nerve growth factor receptor (NGFR), FGF1, OLFML1, and EPGN were upregulated and 98 transcripts such as ACAN, RUNX2, and C21orf96 were downregulated. These upand downregulated genes in trilineage differentiated hNTSCs are listed in Tables 1-3 and online suppl. Table 1; see www.karger.com/doi/10.1159/000516016 for all online suppl. material, (fold change in expression). Ad- 

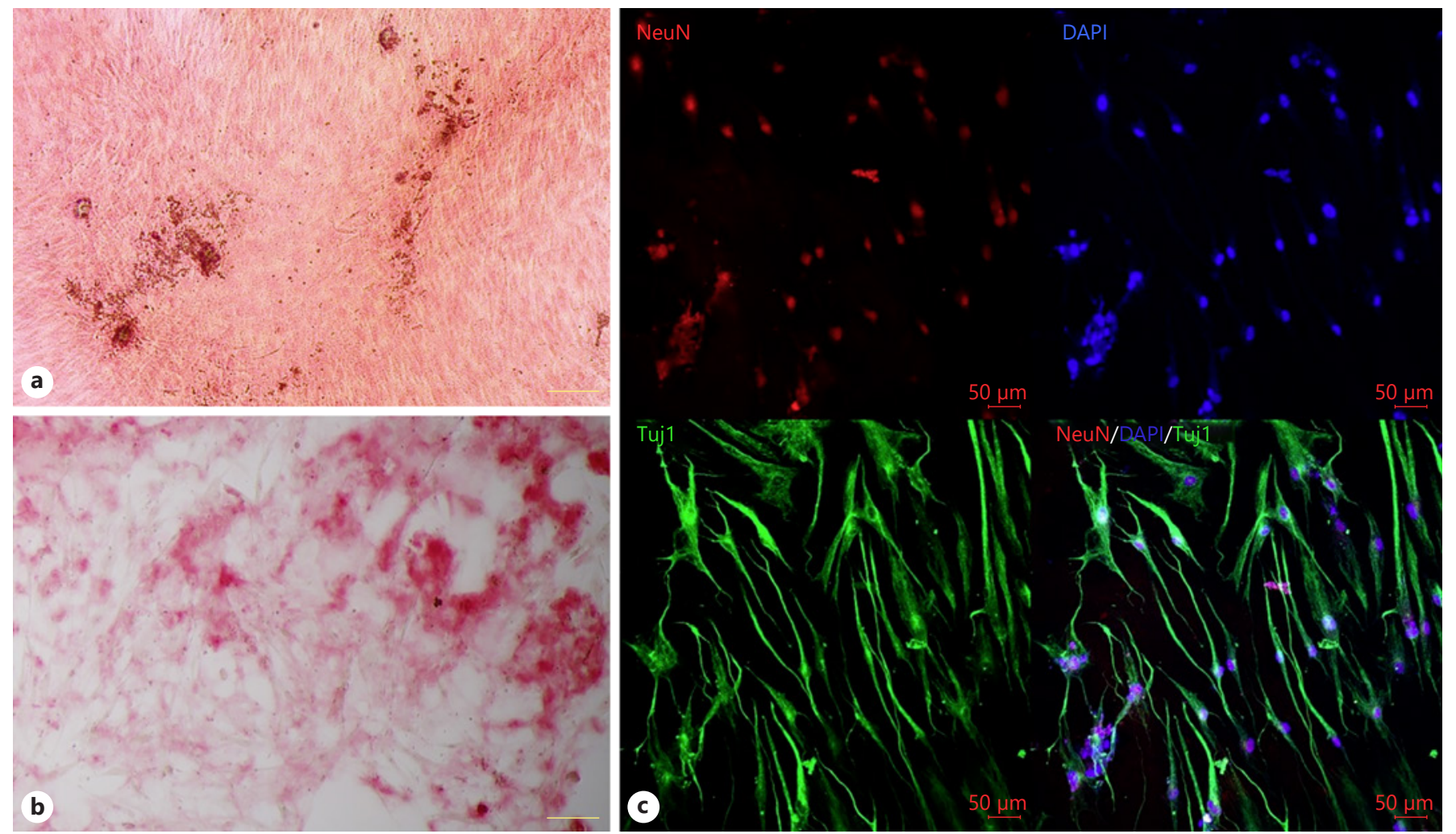

Fig. 2. Histologic analysis of hTMSCs $2-3$ weeks after trilineage differentiation. The cells exposed to chondrogenic (a) and osteogenic (b) medium showed direct evidences of proteoglycan and calcium mineralization seen by Safranin O staining and Alizarin Red staining. Cells after neurogenic induction expressed nestin and $\beta$-tubulin (c). hTMSCs, human turbinate mesenchymal stromal cells. ditionally, principal component analysis plot for comparing differences of cell groups according to differentiation and a heatmap for comparing genes differentially expressed in undifferentiated and trilineage differentiated hNTSCs were prepared. Results are shown in Figures 3, 4.

\section{Gene Ontology Analysis}

Major over- and underrepresented gene ontology (GO) classes are shown in Tables 4-6. In neural differentiated hNTSCs, major GO classes of cytokine or chemokine were abundant. In chondrogenic differentiated hNTSCs, catalysis GO terms were overrepresented. In osteogenic differentiated hNTSCs, GO terms related to oxidoreductase were overrepresented.

\section{Discussion}

Results of the present study verified specific gene expression patterns in hNTSCs according to trilineage differentiation. These results were compared to expression profiles of undifferentiated hNTSCs as a reference. Expression profiles of osteo-, chondro-, and neuro-differentiated hNTSCs were found to be different from each other. Based on expression profile analysis, many genes were shown to be up- or downregulated. Furthermore, GO analyses of regulated gene products showed over- or underexpressed GO classes. These results provide a first step for revealing core molecules associated with characteristics of hNTSCs.

Microarrays have become vital tools for detecting the expression of thousands of genes and for identifying differentially expressed genes. They enable us to explain and compare gene expression patterns of cells during or after differentiation. Based on microarray data, we can comprehend mechanisms controlling the expression of individual cells' characteristics. Some microarray studies have been conducted to check new specific markers and investigate the origin of various diseases including cancers [6-8].

In the otolaryngologic part, partial turbinate resection is one of the most popular and efficient surgery for reduc- 
Table 1. Identification of genes differentially expressed during chondrogenesis

\begin{tabular}{ll}
\hline RefSeq ID & Gene name \\
\hline Upregulated genes & \\
NC_000014.9 & Nudix-type motif 14 \\
NC_000007.14 & Carboxypeptidase A4 \\
NC_000005.10 & HBEGF \\
\hline
\end{tabular}

\section{Downregulated genes}

NC_000009.12 Prostaglandin-endoperoxide synthase 1 (prostaglandin G/H synthase and cyclooxygenase)

NC_000012.12 C-type lectin domain family 2 member D

NC_000077.6 SH3 and cysteine-rich domain 2

NC_000019.8 Hypothetical protein LOC100128567

NC_000010.11 Tet methylcytosine dioxygenase 1

NC_000003.12 Ceruloplasmin (ferroxidase)

NC_000007.14 Hypoxia inducible lipid droplet associated

AK094436.1 Uncharacterized LOC284219

NP_775819.1 Ensembl 59 human genes, GRCh37 assembly-ZNF584

NC_000007.14 Leptin

XM_001123368 Similar to cholinergic receptor, muscarinic 3

NC_000016.10 Proline-rich transmembrane protein 2

NC_000006.12 POU class 5 homeobox 1

NC_000005.10 Family with sequence similarity 153, member B

NC_000005.10 NLR family, apoptosis inhibitory protein

NC_000006.12 Forkhead box Q1

NC_000005.10 Liver-expressed antimicrobial peptide 2

NC_000014.9

NC_000019.10

NC_000014.7

NC_000022.11

NC_000007.14

NC_000007.14

NC_000022.11

FBJ murine osteosarcoma viral oncogene homolog

Intercellular adhesion molecule 5, telencephalin

Uncharacterized LOC440200

Galactose-3-O-sulfotransferase 1

CCZ1 homolog B, vacuolar protein trafficking and biogenesis associated

NC_000005.10

Leucine-rich single-pass membrane protein 1

NC_000010.11

Uncharacterized LOC729461

Phosphodiesterase 8B

NC_000009.11

RUN and FYVE domain containing 2

NC_000019.10

Uncharacterized MGC24103

NC_000011.10

Zinc finger protein 615

NC_000011.10

H19, imprinted maternally expressed transcript

NC_000016.10

KCNQ1 opposite strand/antisense transcript 1

NC_000011.10

Guanine nucleotide binding protein

NC_000020.11

NC_000017.11

Mucin 5AC, oligomeric mucus/gel-forming

NC_000001.11

NC_000009.12

Family with sequence similarity 65 , member C

Ring finger protein 112

Phosphodiesterase 4B, cAMP-specific

NC_000010.9

Carbonic anhydrase IX

NC_000017.11

Uncharacterized protein BC008131

NC_000002.12

RAS, dexamethasone-induced 1

NC_000006.12

Ankyrin repeat domain 36B

NC_000007.14

Tenascin XB

AL110203.1

WD repeat domain 86

NC_000010.11

Uncharacterized LOC158863

NC_000007.14

Uncharacterized LOC399715

NC_000009.12

Nicotinamide phosphoribosyltransferase

NC_000006.12

NC_000017.11

Uncharacterized protein PRO2852

NC_000001.11

Suppressor APC domain containing 1

Gasdermin B

NC_000015.10

Prostaglandin-endoperoxide synthase 2 (prostaglandin G/H synthase and cyclooxygenase)

NC_000004.10

Long intergenic nonprotein coding RNA 597

Uncharacterized LOC100131829 
Table 1 (continued)

\begin{tabular}{ll}
\hline RefSeq ID & Gene name \\
\hline NC_000006.12 & Tudor domain containing 6 \\
NC_000007.14 & Cyclin D binding myb-like transcription factor 1 \\
NC_000002.11 & Uncharacterized LOC100128563 \\
NC_000006.12 & Wilms tumor 1-associated protein \\
NC_000011.10 & Dopamine receptor D4 \\
NC_000020.10 & Uncharacterized LOC29053 \\
NC_000023.11 & Apelin \\
NC_000003.12 & Sarcolemma-associated protein \\
BX647230.1 & Uncharacterized LOC399832 \\
NC_000002.12 & Frizzled-related protein \\
NC_000019.10 & Cartilage intermediate layer protein 2 \\
NC_000008.11 & Metastasis suppressor 1 \\
NC_000014.9 & RAS (RAD and GEM)-like GTP binding 2 \\
\hline
\end{tabular}

Nudix, nucleoside diphosphate-linked moiety X; HBEGF, heparin-binding epidermal growth factor-like growth factor.
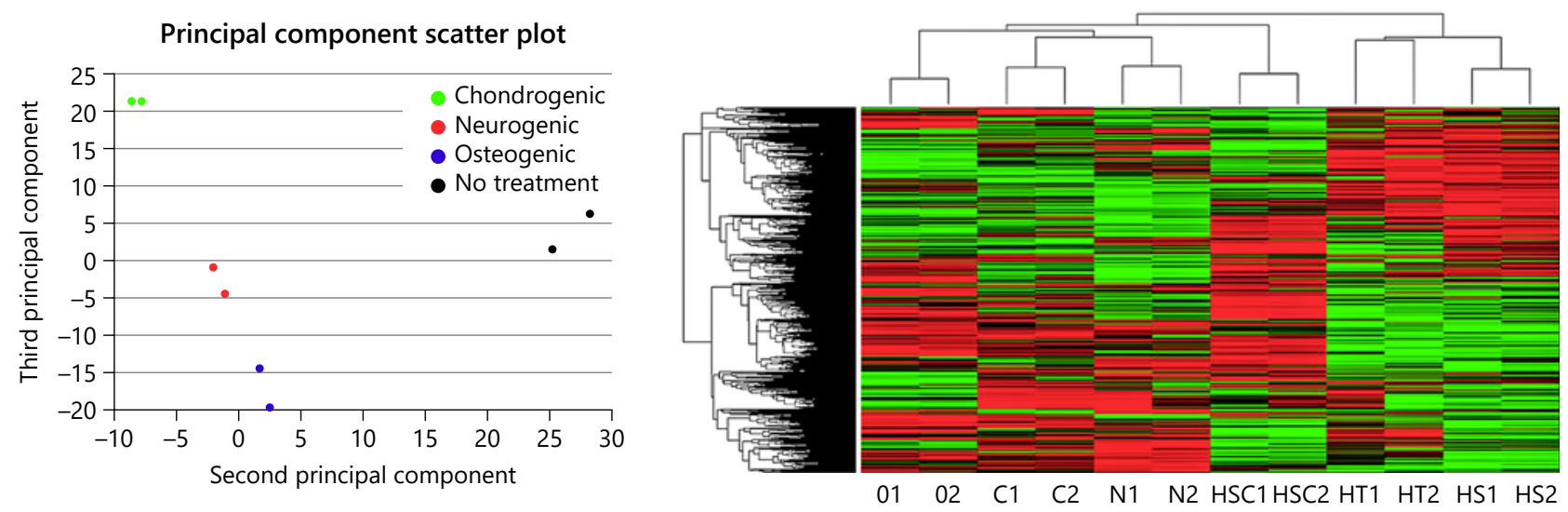

Fig. 3. PCA plot. A PCA plot showed the differences for cell groups according to differentiation. Each spot represented a single array sample. PCA, principal component analysis.

ing nasal symptoms. MSCs derived from the inferior tubinate can be obtained in ease and abundance. Due to this feature, hNTSCs could serve as important resources of adult stem cells. However, characteristics and differentiation of hNTSCs using genome-wide research techniques have not been reported yet. Studies on MSC derived from different tissues have shown a phenotype analogous to each other. Nevertheless, considerable differences in the molecular phenotype among MSCs from different tissues have been reported, showing ontological and functional differences [9]. Thus, it would be important to perform genome-wide gene expression pro- filing and GO analysis for hNTSCs. Thus, this is the first study to obtain gene expression profiles of hNTSCs through microarray.

HBEGF is a mitogenic and chemotactic molecule related to tissue repair and other tissue-modeling phenomena. Krampera et al. [10] have found that HBEGF can increase the proliferation of bone marrow MSCs but prevent chondrogenic differentiation reversibly. However, in our study, hNTSCs showed increasing HBEGF expression during chondrogenic differentiation. Transforming growth factor beta can induce extracellular matrix proteins during chondrogenic differentiation in vitro. In 
Table 2. Identification of genes differentially expressed during osteogenesis

\begin{tabular}{|c|c|}
\hline RefSeq ID & Gene name \\
\hline \multicolumn{2}{|c|}{ Upregulated genes } \\
\hline NC_000009.12 & Mmicroseminoprotein, prostate associated \\
\hline NC_000020.11 & E2F transcription factor 1 \\
\hline NC_000008.11 & Scavenger receptor class $\mathrm{A}$, member 5 \\
\hline NC_000019.10 & Activating transcription factor 5 \\
\hline NC_000002.12 & LY6/PLAUR domain containing 1 \\
\hline NC_000015.10 & Aldehyde dehydrogenase 1 family, member A3 \\
\hline NC_000003.12 & Family with sequence similarity 107, member A \\
\hline NC_000012.12 & DEAD (Asp-Glu-Ala-Asp) box polypeptide 54 \\
\hline NC_000002.12 & Vitrin \\
\hline NC_000011.10 & Matrix metallopeptidase 1 \\
\hline NC_000012.12 & G protein-coupled receptor, class C, group 5, member A \\
\hline NC_000016.10 & Periplakin \\
\hline NC_000010.11 & Interferon-induced protein with tetratricopeptide repeats 3 \\
\hline NC_000017.11 & Chemokine (C-X-C motif) ligand 16 \\
\hline NC_000007.14 & Carboxypeptidase A4 \\
\hline NC_000007.14 & Aldo-keto reductase family 1 , member B10 (aldose reductase) \\
\hline NC_000023.11 & Retinoic acid-induced 2 \\
\hline NC_000006.12 & Transcription factor AP-2 beta (activating enhancer binding protein 2 beta) \\
\hline NC_000014.9 & Deiodinase, iodothyronine, type II \\
\hline NC_000007.14 & STEAP family member 4 \\
\hline NC_000019.10 & Aldehyde dehydrogenase 16 family, member A1 \\
\hline NC_000009.12 & Phytanoyl-CoA dioxygenase domain-containing 1 \\
\hline NC_000023.11 & Nuclear receptor subfamily 0 , group B, member 1 \\
\hline
\end{tabular}

Downregulated genes

NC_000009.12

NC_000001.11

NC_000008.11

NC_000002.12

NC_000007.14

NC_000020.11

NC_000007.14

NC_000001.11

NC_000009.12

NC_000009.12

NC_000005.10

NC_000014.7

NC_000012.12

NC_000017.11

NC_000001.11

NC_000017.11

NC_000012.12

NC_000017.11

NC_000011.10

NC_000006.12

Carbonic anhydrase IX

Protein tyrosine phosphatase, receptor type, f polypeptide (PTPRF), interacting protein (liprin), alpha 4

Hyaluronan synthase 2

Collagen, type IV, alpha 4

Leptin

Fer-1-like family member 4, pseudogene (functional)

Hypoxia inducible lipid droplet-associated

Dual specificity phosphatase 5 pseudogene 1

Very low-density lipoprotein receptor

Prostaglandin D2 synthase $21 \mathrm{kDa}$ (brain)

Family with sequence similarity 153 , member B

Uncharacterized LOC440200

Cysteine and glycine-rich protein 2

Serine/arginine-rich splicing factor 1

Thioredoxin interacting protein

Arachidonate 15-lipoxygenase, type B

C-type lectin domain family 2, member D

Asialoglycoprotein receptor 1

KCNQ1 opposite strand/antisense transcript 1 (nonprotein coding)

Collagen, type XXI, alpha 1 
Table 2 (continued)

\begin{tabular}{ll}
\hline RefSeq ID & Gene name \\
\hline NC_000023.11 & Apelin \\
NC_000006.12 & Vascular endothelial growth factor A \\
NC_000017.11 & Ring finger protein 112 \\
NC_000015.10 & Prader Willi/Angelman region RNA, SNRPN neighbor \\
NC_000001.11 & Uncharacterized LOC100131564 \\
NC_000004.12 & Chromosome 4 open reading frame 47 \\
NC_000002.12 & Ankyrin repeat domain 36B \\
NC_000009.12 & Prune homolog 2 (Drosophila) \\
NC_000013.11 & Periostin, osteoblast-specific factor \\
NC_000022.11 & Galactose-3-O-sulfotransferase 1 \\
NC_000006.12 & Wilms tumor 1-associated protein \\
NC_000019.10 & Cartilage intermediate layer protein 2 \\
NC_000019.10 & Intercellular adhesion molecule 5, telencephalin \\
NC_000021.9 & Junctional adhesion molecule 2 \\
\hline
\end{tabular}

Nudix, nucleoside diphosphate-linked moiety X.

Fig. 4. Gene expression microarray analysis of hTMSCs according to differentiation. Heatmap comparing the genes differently expressed in the groups. Each row and columns matched with a transcript and sample, respectively. Color changed from green, for the lowest degree of expression, to red for the highest degree of expression. hTMSCs, human turbinate mesenchymal stromal cells.

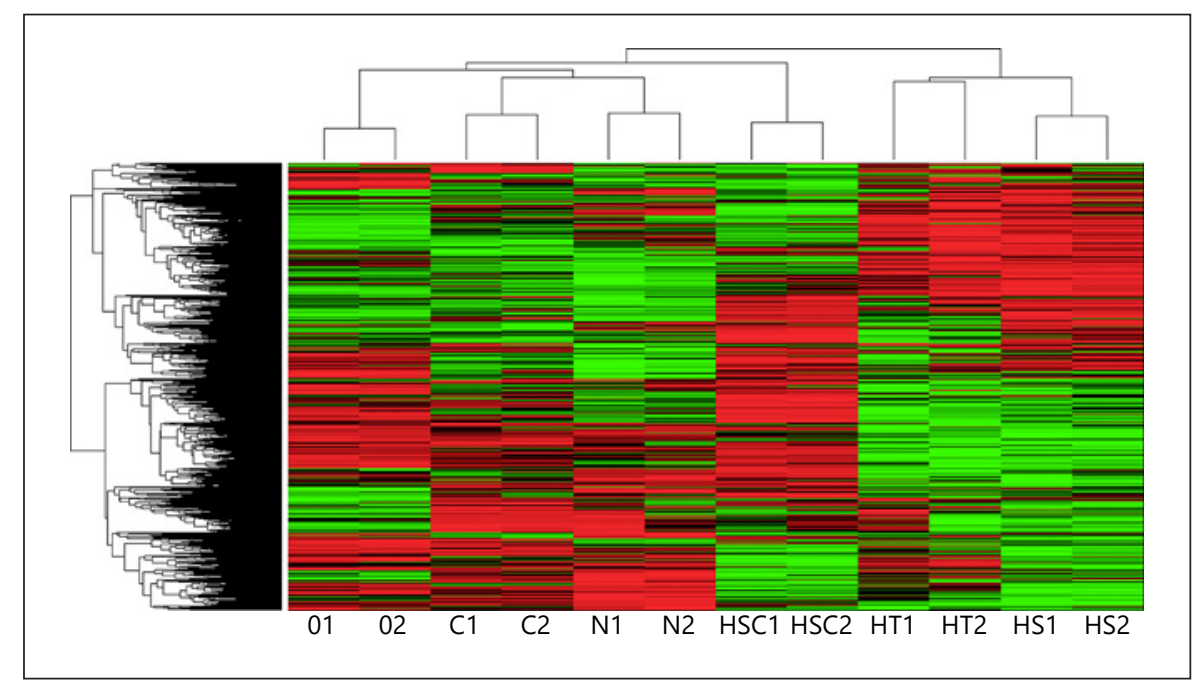

chondrocyte progenitor cells stimulated by transforming growth factor beta, HBEGF was strongly expressed during induction, suggesting that HBEGF could be important in extracellular matrix manufacture as with cell function [11]. Our results showed that hTMSCs after differentiation were most similar to chondrocyte progenitor cells. This could explain why HBEGF is an important role during the chondrogenic differentiation of hNTSCs. ATF5 is a member of the ATF/CREB family of beta-ZIP transcription factors. It is strongly expressed in various neoplasms. It also controls stem cell functions [12]. During osteogenic differentiation of MSCs from human adipose tissue, ATF5 was highly expressed, reaching a peak of expression at the stage of bone mineralization. Vicari et al. [13] have suggested that ATF5 could play an interesting regulatory role during osteogenesis. These results were similar to our results showing that ATF5 was expressed highly during osteogenic differentiation.

E2F1 functions as a DNA-binding domain of $\mathrm{pRb}$. Signaling via the interaction of $\mathrm{pRb}-\mathrm{E} 2 \mathrm{~F}$ is considered to be associated with cell cycle regulation, cell fate, and differentiation of MSCs [14]. pRB is an activator of tissue-specific gene expression along diverse lineages including osteoblast differentiation [15]. In addition, the transcription start site of the ATF5 gene with promoter activity retaining has potential binding sites for several transcrip- 
Table 3. Identification of genes differentially expressed during neurogenesis

\begin{tabular}{|c|c|}
\hline RefSeq ID & Gene name \\
\hline \multicolumn{2}{|c|}{ Upregulated genes } \\
\hline NC_000002.12 & LON peptidase $\mathrm{N}$-terminal domain and ring finger 2 \\
\hline NC_000004.12 & Annexin A10 \\
\hline NC_000004.12 & Heparan sulfate (glucosamine) 3-O-sulfotransferase 1 \\
\hline NC_000002.12 & Adapter-related protein complex 1 , sigma 3 subunit \\
\hline NC_000005.10 & Family with sequence similarity 169, member A \\
\hline NC_000009.12 & TEK tyrosine kinase, endothelial \\
\hline NC_000007.14 & Insulin-like growth factor binding protein 1 \\
\hline NC_000017.11 & Chemokine (C-X-C motif) ligand 16 \\
\hline NC_000007.14 & Fibrinogen-like 2 \\
\hline NC_000004.12 & Chemokine (C-X-C motif) ligand 6 \\
\hline NC_000005.10 & Proprotein convertase subtilisin/kexin type 1 \\
\hline NC_000012.12 & Parathyroid hormone-like hormone \\
\hline NC_000001.11 & Lipid phosphate phosphatase-related protein type 4 \\
\hline NC_000004.12 & Chemokine (C-X-C motif) ligand 1 (melanoma growth stimulating activity, alpha) \\
\hline NC_000017.11 & Netrin 1 \\
\hline NC_000012.12 & Retinol dehydrogenase 5 (11-cis/9-cis) \\
\hline NC_000011.10 & Chordin-like 2 \\
\hline NC_000001.11 & Hydroxysteroid (11-beta) dehydrogenase 1 \\
\hline NC_000005.10 & Cytoplasmic FMR1 interacting protein 2 \\
\hline NC_000002.12 & Sperm-associated antigen 16 \\
\hline NC_000004.12 & Chemokine (C-X-C motif) ligand 2 \\
\hline NC_000009.12 & Tumor necrosis factor (ligand) superfamily, member 15 \\
\hline NC_000009.12 & Microseminoprotein, prostate associated \\
\hline NC_000001.11 & Regulatory subunit of type II PKA R-subunit (RIIa) domain containing 1 \\
\hline NC_000012.12 & Serine dehydratase-like \\
\hline NC_000002.12 & Kynureninase \\
\hline NC_000017.11 & Chemokine (C-C motif) ligand 7 \\
\hline NC_000011.10 & Olfactomedin-like 1 \\
\hline NC_000003.12 & Acyl-CoA oxidase 2, branched chain \\
\hline NC_000020.11 & Potassium voltage-gated channel, modifier subfamily S, member 1 \\
\hline NC_000010.11 & Internexin neuronal intermediate filament protein, alpha \\
\hline NC_000004.12 & Epithelial mitogen \\
\hline NC_000014.9 & Long intergenic nonprotein coding RNA 643 \\
\hline NC_000001.11 & Chitinase 3-like 2 \\
\hline NC_000003.12 & Claudin 1 \\
\hline NC_000013.11 & Glypican 5 \\
\hline NC_000014.9 & Somatostatin receptor 1 \\
\hline NC_000017.11 & Nerve growth factor receptor \\
\hline NC_000017.11 & Family with sequence similarity 20 , member A \\
\hline NC_000012.12 & Spexin hormone \\
\hline NC_000017.11 & Chemokine (C-C motif) ligand 2 \\
\hline NC_000007.14 & Tissue factor pathway inhibitor 2 \\
\hline NC_000016.10 & Chromosome 16 open reading frame 89 \\
\hline NC_000004.12 & Chemokine (C-X-C motif) ligand 3 \\
\hline NC_000007.14 & Carboxypeptidase A4 \\
\hline NC_000007.14 & Calcium channel, voltage-dependent, alpha $2 /$ delta subunit 1 \\
\hline NC_000003.12 & Solute carrier family 7 , member 14 \\
\hline NC_000001.11 & Vascular cell adhesion molecule 1 \\
\hline NC_000018.10 & Collectin sub-family member 12 \\
\hline NC_000005.10 & Fibroblast growth factor 1 (acidic) \\
\hline NC_000011.10 & Dedicator of cytokinesis 1 pseudogene \\
\hline NC_000012.12 & TAP binding protein-like \\
\hline NC_000004.12 & TLR3 \\
\hline NC_000011.10 & Midkine (neurite growth-promoting factor 2) \\
\hline
\end{tabular}


Table 3 (continued)

\begin{tabular}{|c|c|}
\hline RefSeq ID & Gene name \\
\hline NC_000010.11 & Interferon-induced protein with tetratricopeptide repeats 3 \\
\hline NC_000008.11 & Musculin \\
\hline NC_000020.11 & Peptidase inhibitor 3, skin-derived \\
\hline NC_000007.14 & Interleukin 6 \\
\hline \multicolumn{2}{|c|}{ Downregulated genes } \\
\hline NC_000011.10 & Insulin-like growth factor 2 \\
\hline NC_000010.11 & Zinc finger protein 91 pseudogene \\
\hline NC_000001.11 & Glutamate-ammonia ligase \\
\hline NC_000011.10 & H19, imprinted maternally expressed transcript (nonprotein coding) \\
\hline NC_000012.12 & Taste receptor, type 2 , member 30 \\
\hline NC_000015.10 & Aggrecan \\
\hline NC_000016.10 & Metallothionein 1X \\
\hline NC_000009.12 & Uncharacterized protein PRO2852 \\
\hline NC_000007.14 & Leptin \\
\hline NC_000003.12 & Sarcolemma associated protein \\
\hline NC_000017.11 & Ankyrin repeat and FYVE domain containing 1 \\
\hline NC_000005.10 & Family with sequence similarity 153 , member B \\
\hline NC_000001.11 & Cell adhesion molecule 3 \\
\hline NC_000019.10 & Cartilage oligomeric matrix protein \\
\hline NC_000013.11 & Collagen alpha-1(II) chain-like \\
\hline AB007954.1 & Uncharacterized LOC57235 \\
\hline NC_000022.11 & POM121 transmembrane nucleoporin-like 8 pseudogene \\
\hline NC_000006.12 & FK506 binding protein 5 \\
\hline NC_000020.11 & Junctophilin 2 \\
\hline NC_000007.14 & Pyruvate dehydrogenase kinase, isozyme 4 \\
\hline NC_000017.11 & Hepatic leukemia factor \\
\hline NC_000019.10 & Cytokine receptor-like factor 1 \\
\hline NC_000008.11 & Hair growth associated \\
\hline NC_000017.11 & Noggin \\
\hline NC_000010.11 & Tet methylcytosine dioxygenase 1 \\
\hline NC_007869.1 & Chemokine (C-X-C motif) receptor 7 \\
\hline NC_000010.11 & Calcium channel, voltage-dependent, beta 2 subunit \\
\hline NC_000017.11 & Chromosome 17 open reading frame 67 \\
\hline NC_000010.11 & Sorbin and $\mathrm{SH} 3$ domain containing 1 \\
\hline NC_000017.11 & Arachidonate 15-lipoxygenase, type B \\
\hline NC_000001.11 & HNRNPU antisense RNA 1 \\
\hline NC_000023.11 & TSC22 domain family, member 3 \\
\hline NC_000022.11 & POM121 transmembrane nucleoporin-like 1, pseudogene \\
\hline NC_000002.12 & Frizzled-related protein \\
\hline NC_000002.12 & Methyltransferase like 21A \\
\hline NC_000006.12 & POU class 5 homeobox 1 \\
\hline NC_000004.10 & Uncharacterized LOC100131829 \\
\hline NC_000014.9 & Serpin peptidase inhibitor, clade A (alpha- 1 antiproteinase, antitrypsin), member 5 \\
\hline NC_000010.11 & Nebulette \\
\hline NC_000013.11 & Regulator of cell cycle \\
\hline NC_000017.11 & Coronin 6 \\
\hline NC_000006.12 & Wilms tumor 1 associated protein \\
\hline NC_000011.10 & Platelet derived growth factor D \\
\hline NC_000021.9 & RUNX1 intronic transcript 1 \\
\hline AL110203.1 & Uncharacterized LOC158863 \\
\hline NC_000007.1 & SND1 intronic transcript 1 \\
\hline NC_000017.11 & Serine/arginine-rich splicing factor 1 \\
\hline NC_000008.11 & PHD finger protein 20-like 1 \\
\hline NC_000011.10 & Zinc finger and BTB domain containing 16 \\
\hline NC_000006.12 & MyoD family inhibitor \\
\hline
\end{tabular}


Table 3 (continued)

\begin{tabular}{ll}
\hline RefSeq ID & Gene name \\
\hline NC_000007.14 & WD repeat domain 86 \\
NC_000020.11 & Inhibitor of DNA binding 1, dominant negative helix-loop-helix protein \\
NC_000020.11 & Acyl-CoA synthetase short-chain family member 1 \\
NC_000009.11 & Uncharacterized MGC24103 \\
NC_000005.10 & CD14 molecule \\
NC_000015.10 & Putative ubiquitin-conjugating enzyme E2Q2-like protein \\
NC_000020.11 & V-maf avian musculoaponeurotic fibrosarcoma oncogene homolog B \\
NC_000006.12 & Runt-related transcription factor 2 \\
NC_000007.14 & Leucine-rich single-pass membrane protein 1 \\
NC_000011.10 & BH3-like motif containing, cell death inducer \\
NC_000003.12 & Long intergenic nonprotein coding RNA 312 \\
NT_113901.1 & Uncharacterized LOC645566 \\
NC_000005.10 & Liver expressed antimicrobial peptide 2 \\
NC_000014.9 & FBJ murine osteosarcoma viral oncogene homolog \\
NC_000012.12 & Inositol 1,4,5-trisphosphate receptor, type 2 \\
NC_000014.7 & Uncharacterized LOC440200 \\
NC_000016.10 & Copine VII \\
NC_000019.10 & Cartilage intermediate layer protein 2 \\
AK094436.1 & Uncharacterized LOC284219 \\
NC_000022.11 & POM121 transmembrane nucleoporin-like 9, pseudogene \\
NC_000008.11 & ASAP1 intronic transcript 1 \\
NC_000013.11 & Mitochondrial inner membrane organizing system 1 pseudogene 1 \\
NC_000002.12 & Ankyrin repeat domain 36B \\
NC_000022.11 & Galactose-3-O-sulfotransferase 1 \\
NC_000017.11 & RAS, dexamethasone-induced 1 \\
NC_000001.11 & Endothelin 2 \\
\hline
\end{tabular}

Table 4. Major over or underrepresented GO classes during chondrogenesis

\begin{tabular}{|c|c|c|}
\hline Reporter ID & Reporter name & $p$ value \\
\hline \multicolumn{3}{|l|}{ Upregulated } \\
\hline GO:0008768 & UDP-sugar diphosphatase activity & 0.001193246 \\
\hline GO:0047631 & ADP-ribose diphosphatase activity & 0.002623259 \\
\hline GO:0009046 & Zinc D-Ala-D-Ala carboxypeptidase activity & 0.004407889 \\
\hline GO:0061473 & Murein tripeptide carboxypeptidase activity & 0.004407889 \\
\hline GO:0005515 & Protein binding & 0.004645598 \\
\hline GO:0008317 & Gurken receptor binding & 0.005477128 \\
\hline GO:0004180 & Carboxypeptidase activity & 0.00784908 \\
\hline GO:0005154 & Epidermal growth factor receptor binding & 0.008204381 \\
\hline GO:0004181 & Metallocarboxypeptidase activity & 0.008559554 \\
\hline \multicolumn{3}{|l|}{ Downregulated } \\
\hline GO:0004118 & cGMP-stimulated cyclic-nucleotide phosphodiesterase activity & 0.000558946 \\
\hline GO:0004120 & Photoreceptor cyclic-nucleotide phosphodiesterase activity & 0.000558946 \\
\hline GO:0004119 & cGMP-inhibited cyclic-nucleotide phosphodiesterase activity & 0.000593312 \\
\hline GO:0004117 & Calmodulin-dependent cyclic-nucleotide phosphodiesterase activity & 0.000628668 \\
\hline GO:0004115 & $3^{\prime}, 5^{\prime}$-cyclic-AMP phosphodiesterase activity & 0.000740648 \\
\hline GO:0047555 & $3^{\prime}, 5^{\prime}$-cyclic-GMP phosphodiesterase activity & 0.000740648 \\
\hline GO:0004114 & $3^{\prime}, 5^{\prime}$-cyclic-nucleotide phosphodiesterase activity & 0.000861435 \\
\hline GO:0004112 & Cyclic-nucleotide phosphodiesterase activity & 0.001539249 \\
\hline GO:0004130 & Cytochrome-c peroxidase activity & 0.001946968 \\
\hline
\end{tabular}

GO, gene ontology. 
Table 5. Identification of genes differentially expressed during neurogenesis

\begin{tabular}{|c|c|c|}
\hline \multicolumn{3}{|l|}{ Upregulated } \\
\hline GO:0008009 & Chemokine activity & $1.92 \mathrm{E}-08$ \\
\hline GO:0042379 & Chemokine receptor binding & $1.93 \mathrm{E}-08$ \\
\hline GO:0045236 & CXCR chemokine receptor binding & $1.98 \mathrm{E}-08$ \\
\hline GO:0005125 & Cytokine activity & $1.02 \mathrm{E}-05$ \\
\hline GO:0031725 & CXCR6 chemokine receptor binding & $1.24 \mathrm{E}-05$ \\
\hline GO:0031723 & CXCR4 chemokine receptor binding & $1.36 \mathrm{E}-05$ \\
\hline GO:0031724 & CXCR5 chemokine receptor binding & $2.82 \mathrm{E}-05$ \\
\hline GO:0031732 & CCR7 chemokine receptor binding & $2.94 \mathrm{E}-05$ \\
\hline \multicolumn{3}{|c|}{ Downregulated } \\
\hline GO:0010314 & Phosphatidylinositol-5-phosphate binding & 0.001318916 \\
\hline GO:0001010 & Sequence-specific DNA binding transcription factor recruiting transcription factor activity & 0.002113322 \\
\hline GO:0001130 & Sequence-specific DNA binding bacterial-type RNA polymerase transcription factor activity & 0.002113322 \\
\hline GO:0001142 & Sequence-specific DNA binding mitochondrial RNA polymerase transcription factor activity & 0.002113322 \\
\hline GO:0001167 & Sequence-specific DNA binding RNA polymerase I transcription factor activity & 0.002113322 \\
\hline GO:0001199 & Metal ion regulated sequence-specific DNA binding transcription factor activity & 0.002113322 \\
\hline
\end{tabular}

Table 6. Identification of genes differentially expressed during osteogenesis

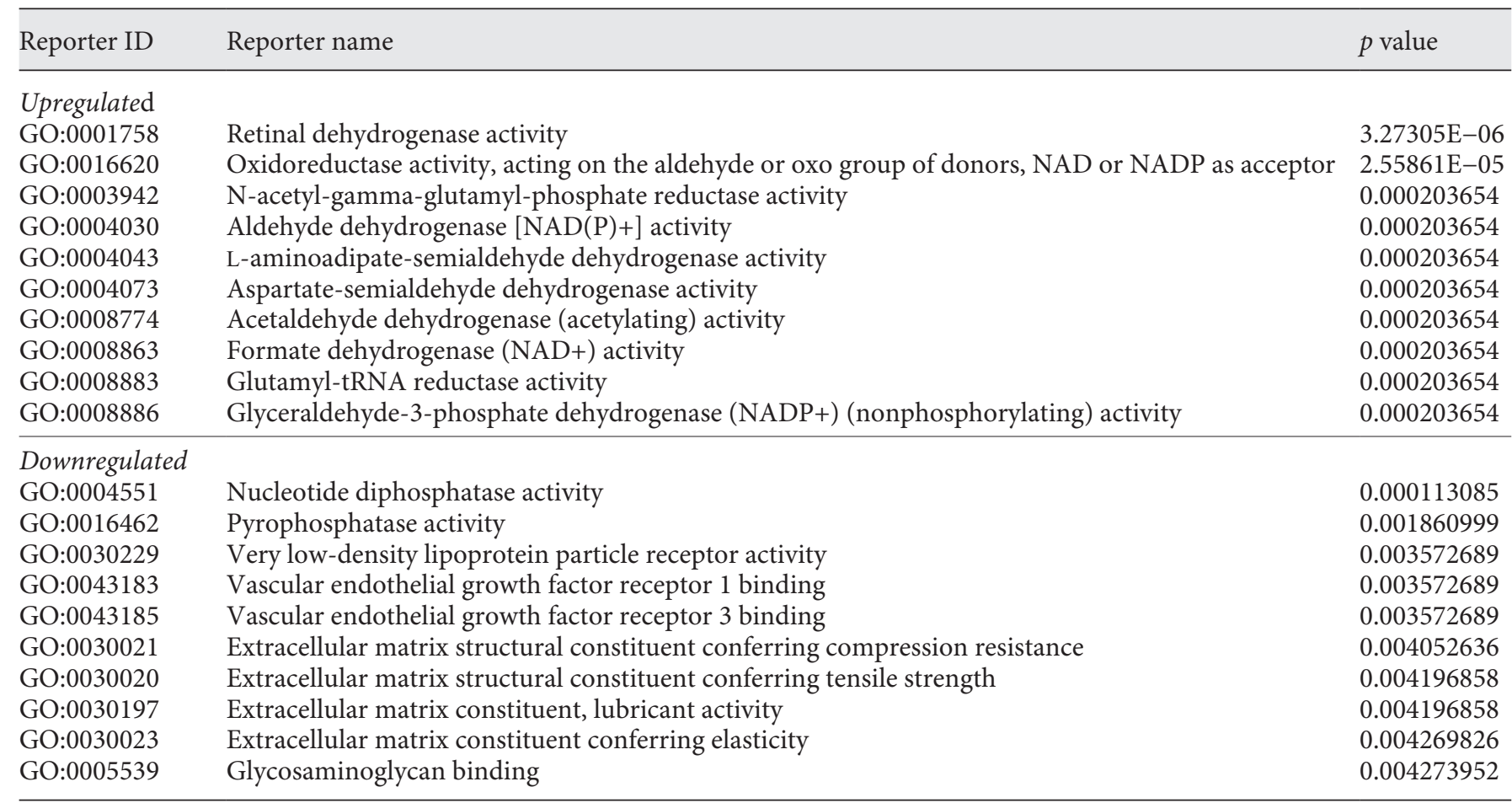


tion factors, including EBF1, Sp1, and E2F1. In particular, mutation of the E2F1-binding site obviously impairs the activity of the ATF5 promoter, showing that this site is the principal cis-element for the transcriptional activation of human ATF5 gene [12]. These facts could support our results showing that E2F1 was expressed highly during induction for osteogenic differentiation. IGFBP coordinate and regulate biological activities of IGF, a powerful neurotrophic factor that provokes proliferation, migration, and differentiation of glial and neuronal cells and prevents these cells from going through apoptosis [16]. Some authors have proposed that MSCs might have neurotrophic properties to differentiate into neuron and repair nerve. A previous study using a nerve injury model has shown a considerable increment in the release of IGFBP-1 from transplanted MSCs and IGFBP-1 during repair of peripheral nerves has significant influence on engraftment and neural differentiation of transplanted cells. Therefore, it has been suggested that IGFBP-1 is an essential neurotrophic factor released from MSCs that can promote regeneration of damaged neural tissues [17]. NGFRs are a group of growth factor receptors that can specifically bind to neurotrophins. They are also used as cell surface markers to define MSC phenotype [18]. In particular, in several studies using monoclonal antibodies to NGFR for isolation of NGFR positive MSCs, this subset of cells has a higher neuronal differentiation property than the whole MSCs population $[19,20]$. These results were similar to our results showing that IGFBP-1 and NGFR were expressed highly during induction for neurogenic differentiation.

GO is a method to comprehend massive-scale gene expression data. In the GO database, top-level ontologies are molecular function, biological process, and cellular component [21]. In our study, according to GO analysis, GO terms related to molecular functions were overrepresented in all trilineage differentiation of hNTSCs. However, GO terms related to cytokine or chemokine were overrepresented in neural differentiated hNTSCs while GO terms related to catalysis and GO terms related to oxidoreductase activity were mainly overrepresented in chondrogenic and osteogenic differentiated hNTSCs, respectively. These facts clearly showed that differently differentiated MSCs had other specific characteristics. In the future, further studies are needed to find the meaning of other major overrepresented GO terms in differentiated hNTSCs.

We performed microarray analysis during trilineage differentiation of hNTSCs in vitro. We found that over 300 genes were expressed differentially in hNTSCs during differentiation. However, certain limitations need to be noted in this study. Of over 300 genes in hNTSCs we found, we could not evaluate characteristics of the majority of genes regulated during in vitro differentiation. Additional studies are needed to determine changes of most genes detected by microarray analysis and characterize these genes more precisely. Additionally, it would be important to identify sequential gene expression during differentiation. Previously, temporal changes of cell growth and osteoblast phenotype-associated genes in human and rodent models have been reported $[22,23]$. In future, we will evaluate sequential expression profiling of hNTSCs during differentiation. However, gene expression profiling and GO analysis of this study are useful for defining genes responsible for characteristics of hNTSCs and for analysis of responses of hNTSCs to signals that drive different differentiation processes. Our results may provide novel information of genes involved in the differentiation of hNTSCs.

\section{Conclusions}

We described genome-wide gene expression patterns of hNTSCs during trilineage differentiation. Differences in expression profiles of trilineage differentiated hNTSCs were found and 316 candidate genes were shown to be definitely up- or downregulated. Further analytical studies should be conducted to comprehensively determine functional roles of these differentially expressed genes detected by microarray analysis. In the future, gene expression data of hNTSCs could offer important understanding for cellular differentiation processes in MSCs.

\section{Statement of Ethics}

This study was approved by the institutional review board committee (IRB No. KC08TISS0341), and informed consent was obtained from all patients.

\section{Conflict of Interest Statement}

The authors have no conflicts of interest to declare.

\section{Funding Sources}

This work was supported by the Korea Health Industry Development Institute funded by the Ministry of Health and Welfare (HI14C3228) and the Bio \& Medical Technology Development Program of the National Research Foundation (NRF) 
funded by the Korean government (MSIT) (No. NRF2019M3A9H2032424, No. NRF-2019M3E5D5064110). This research was supported by the Basic Science Research Program through the National Research Foundation of Korea (NRF) grant funded by the Ministry of Education (2018R1D1A1B07045421). This work was supported by the Basic Science Research Program through the National Research Foundation of Korea (NRF) funded by the Ministry of Education (2017R1D1A1B03027903). This research was supported by the Bio \& Medical Technology Development Program of the National Research Foundation (NRF) funded by the Korean government (MSIT) (No. NRF-2018M3A9E8020856). This research was supported by the Basic Science Research Program through a National Research Foundation of Korea (NRF) grant funded by the Ministry of Education (2017R1D1A1B03034868). This work was also supported by the Institute of Clinical Medicine Research of Bucheon St. Mary's Hospital, Research Fund, 2018, and this study was supported by a grant of the E.N.T. Fund of the Catholic University of Korea made in the program year of 2017 and 2018. The sponsors had no role in study design, data collection and analysis, decision to publish, or preparation of the manuscript.

\section{Author Contributions}

Sun Hwa Park: data analysis, drafting, final approval, and accountability for all aspects of the work; Do Hyun Kim: data analysis, drafting, final approval, and accountability for all aspects of the work; Mi Hyun Lim: data analysis, drafting, final approval, and accountability for all aspects of the work; Sang A Back: data analysis, drafting, final approval, and accountability for all aspects of the work; Byeong Gon Yun: data analysis, drafting, final approval, and accountability for all aspects of the work; Jung Ho Jeun: data analysis, drafting, final approval, and accountability for all aspects of the work; Jung Yeon Lim: data analysis, drafting, final approval, and accountability for all aspects of the work; Su Young Kim: data analysis, drafting, final approval, and accountability for all aspects of the work; Se Hwan Hwang: data analysis, drafting, final approval, and accountability for all aspects of the work; Sung Won Kim: data analysis, drafting, final approval, and accountability for all aspects of the work.

\section{References}

1 Hwang SH, Kim SY, Park SH, Choi MY, Kang HW, Seol YJ, et al. Human inferior turbinate: an alternative tissue source of multipotent mesenchymal stromal cells. Otolaryngol Head Neck Surg. 2012 Sep;147(3):568-74.

2 Berger G, Balum-Azim M, Ophir D. The normal inferior turbinate: histomorphometric analysis and clinical implications. Laryngoscope. 2003 Jul;113(7):1192-8.

3 Hwang SH, Kim SY, Park SH, Choi MY, Back SA, Kim YI, et al. Osteogenic differentiation of human turbinate mesenchymal stromal cells. J Tissue Eng Regen Med. 2011 Dec;8(6): 544-53.

4 Hwang SH, Kim SW, Kim SY, Park SH, Lee HJ, Choi MY, et al. Human turbinate mesenchymal stromal cells as a potential option for cartilage tissue engineering. J Tissue Eng Regen Med. 2011 Dec;8(6):536-43.

5 Mori G, Brunetti G, Oranger A, Carbone C, Ballini A, Lo Muzio L, et al. Dental pulp stem cells: osteogenic differentiation and gene expression. Ann N Y Acad Sci. 2011 Nov; 1237: $47-52$.

6 Aonuma H, Ogura N, Takahashi K, Fujimoto $\mathrm{Y}$, Iwai S, Hashimoto $\mathrm{H}$, et al. Characteristics and osteogenic differentiation of stem/progenitor cells in the human dental follicle analyzed by gene expression profiling. Cell Tissue Res. 2012 Nov;350(2):317-31.

7 Kim SH, Kim YS, Lee SY, Kim KH, Lee YM, Kim WK, et al. Gene expression profile in mesenchymal stem cells derived from dental tissues and bone marrow. J Periodontal Implant Sci. 2011 Aug;41(4):192-200.

8 Sharov AA, Piao Y, Ko MS. Gene expression profiling of mouse embryos with microarrays. Meth Enzymol. 2010;477:511-41.
9 Al-Nbaheen M, Vishnubalaji R, Ali D, Bouslimi A, Al-Jassir F, Megges M, et al. Human stromal (mesenchymal) stem cells from bone marrow, adipose tissue and skin exhibit differences in molecular phenotype and differentiation potential. Stem Cell Rev. 2013 Feb; 9(1):32-43.

10 Krampera M, Pasini A, Rigo A, Scupoli MT, Tecchio C, Malpeli G, et al. HB-EGF/HER-1 signaling in bone marrow mesenchymal stem cells: inducing cell expansion and reversibly preventing multilineage differentiation. Blood. 2005 Jul 1;106(1):59-66.

11 Takeda $\mathrm{H}$, Inoue $\mathrm{H}$, Kutsuna T, Matsushita N, Takahashi T, Watanabe S, et al. Activation of epidermal growth factor receptor gene is involved in transforming growth factor-betamediated fibronectin expression in a chondrocyte progenitor cell line. Int J Mol Med. 2010 Apr;25(4):593-600.

12 Wei Y, Ge Y, Zhou F, Chen H, Cui C, Liu D, et al. Identification and characterization of the promoter of human ATF5 gene. J Biochem. 2010 Aug;148(2):171-8.

13 Vicari L, Calabrese G, Forte S, Giuffrida R, Colarossi C, Parrinello NL, et al. Potential role of activating transcription factor 5 during osteogenesis. Stem Cells Int. 2016;2016: 5282185.

14 Popov B, Petrov N. pRb-E2F signaling in life of mesenchymal stem cells: cell cycle, cell fate, and cell differentiation. Genes Dis. 2014 Dec; 1(2):174-87.

15 Flowers S, Xu F, Moran E. Cooperative activation of tissue-specific genes by pRB and E2F1. Cancer Res. 2013 Apr 1;73(7):2150-8.

16 Salehi Z, Mashayekhi F, Naji M, Pandamooz S. Insulin-like growth factor-1 and insulin- like growth factor binding proteins in cerebrospinal fluid during the development of mouse embryos. J Clin Neurosci. 2009 Jul; 16(7):950-3.

17 Lee EJ, Xu L, Kim GH, Kang SK, Lee SW, Park $\mathrm{SH}$, et al. Regeneration of peripheral nerves by transplanted sphere of human mesenchymal stem cells derived from embryonic stem cells. Biomaterials. 2012 Oct;33(29):7039-46.

18 Zhang D, Kilian KA. The effect of mesenchymal stem cell shape on the maintenance of multipotency. Biomaterials. 2013 May; 34(16):3962-9.

19 Quirici N, Scavullo C, de Girolamo L, Lopa S, Arrigoni E, Deliliers GL, et al. Anti-L-NGFR and -CD34 monoclonal antibodies identify multipotent mesenchymal stem cells in human adipose tissue. Stem Cells Dev. 2010 Jun; 19(6):915-25.

20 Yamamoto N, Akamatsu H, Hasegawa S, Yamada T, Nakata S, Ohkuma M, et al. Isolation of multipotent stem cells from mouse adipose tissue. J Dermatol Sci. 2007 Oct; 48(1):43-52.

21 Beissbarth T. Interpreting experimental results using gene ontologies. Meth Enzymol. 2006;411:340-52.

22 Kulterer B, Friedl G, Jandrositz A, SanchezCabo F, Prokesch A, Paar C, et al. Gene expression profiling of human mesenchymal stem cells derived from bone marrow during expansion and osteoblast differentiation. BMC Genomics. 2007 Mar 12;8:70.

23 Stein GS, Lian JB. Molecular mechanisms mediating proliferation/differentiation interrelationships during progressive development of the osteoblast phenotype. Endocr Rev. 1993 Aug;14(4):424-42. 